expansions almost all B cells (80\%) had a DN2 phenotype. DN2 cells predominate in African-American patients with active disease and nephritis, anti-Smith and anti-RNA autoantibodies. Among B cells, they express the highest levels of a Tbet/Zeb2 transcriptional network characteristic of effector $T$ cells and lack the negative TLR regulator, TRAF5. Consistent with this transcriptional profile DN2 are hyper-responsive to TLR7. Moreover, DN2 cells share with activated naïve cells phenotypic, functional features, and a very similar transcriptome. When stimulated in vitro DN2 readily differentiated into plasma cells and produced autoantibodies.

Conclusions DN2 and activated naïve represent a separate B cell lineage with a distinct origin and function as they differed from other B cells subsets both in uniquely expressing several genes and a lack expression of other genes. This study defines a distinct differentiation fate of human autoreactive naïve $B$ cells into effector plasma cell precursors with innate hyperresponsiveness to stimuli relevant to SLE, establishes the components and prominence of extrafollicular B cell activation in this disease, and identifies new therapeutic targets.
A

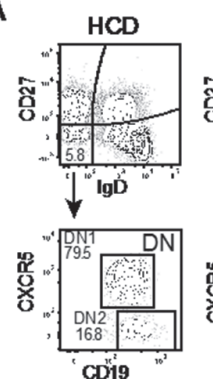

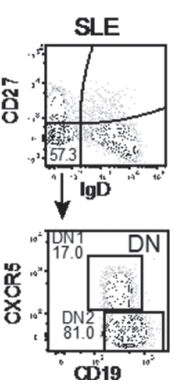

B

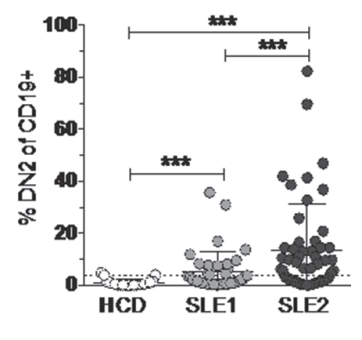

Abstract Al-07 Figure 1 A) representative flow profiles showing that IgD-/CD27-/CXCR5-/CD19++ DN2 B cells are rare in HCD but expanded in many SLE patients. B) the frequency of DN2 for HCD and 2 SLE patient cohorts as percentage of CD19+

\section{AI-08 CHARACTERIZATION OF HUMAN AGE-ASSOCIATED B CELLS (ABCS) IN NORMAL AND LUPUS PERIPHERAL BLOOD}

Jennifer L Barnas*, Jennifer Albrecht, Nida Meednu, Lin Gao, Mary O'Connell, R John Looney, Jennifer H Anolik. University of Rochester, Rochester, NY, USA

\subsection{6/lupus-2018-Ism.8}

Background Age-associated B cells (ABCs) are associated with autoantibody production in lupus-like mice. This population is expanded upon exposure to self-antigen and interferon- $\gamma$. $\mathrm{ABCs}$ in mice are identified as CD11c+, CD21- and T-bet+. However, the characterization of these cells and factors promoting their generation in human SLE is unclear. The purpose of this study was to define ABCs in human SLE patients and their association with interferon (IFN) status.

Methods Peripheral blood leukocytes were purified by density gradient centrifugation from 26 lupus patients with a range of disease activity $(n=10$ with flaring disease, $n=16$ controlled disease) and 6 normal healthy donors. Surface and T-bet intracellular antibody staining was analyzed in a blinded fashion by flow cytometry. A putative population of ABCs was defined as CD3 - CD19+ CD11c+ CD21- cells. Samples were divided into groups regardless of disease status based upon percentage of $\mathrm{B}$ cells with putative $\mathrm{ABC}$ phenotype. B cell subset analysis was completed on the groups expressing minimal $(\leq 2 \%)$, intermediate $(2 \%-7 \%)$, or high $(\geq 8 \%)$ levels of ABCs. In addition, the $\mathrm{B}$ cell subset distribution (switched memory, unswitched memory, total naïve, and $\mathrm{CD} 27-\mathrm{IgD}$ - double negative) and phenotypic markers (CXCR3, T-bet, CD24, IgD, and CD27) of putative $\mathrm{ABCs}$ were assessed. IFN- $\alpha$, IFN- $\beta$, and IFN- $\lambda$ levels were quantitated by ELISA from sera drawn at the same time.

Results Five samples were identified as having high putative ABC levels (range $8.4 \%-20.5 \%$ of B cells, mean 14.9\%). These CD11c+CD21- CD19+CD3 - cells were predominantly CD24-CD27- IgD-. Across all 32 samples, the percentage of putative $\mathrm{ABCs}$ positively correlated with percentage of T-bet $+\mathrm{B}$ cells $\left(\mathrm{r}=0.819, \mathrm{p}=5.4 \times 10^{-9}\right)$, IgD- CD27(double negative) B cells $(\mathrm{r}=0.59, \mathrm{p}=0.0003)$, and CD24double negative cells $\left(r=0.74, p=8.72 \times 10^{-7}\right)$ with a high degree of statistical significance. Paired serum samples had a range of type I and type IIII interferon levels (mean +SEM for all samples): $6.2 \pm 2.1 \mathrm{pg} / \mathrm{m}$ (IFN- $\alpha$ ), $4.0 \pm 0.6 \mathrm{pg} / \mathrm{ml}$ (IFN$\beta$ ), and $44.1 \pm 9.6 \mathrm{pg} / \mathrm{ml}$ (IFN- $\lambda 1)$. Analysis including correlation between $\mathrm{B}$ cell subsets, interferon status, clinical features and disease activity is in progress.

Conclusions An expanded putative ABC population (CD11c+ CD21- CD19+ CD3-) was identified in a subset of human peripheral blood samples. This subset is positively correlated with IgD-CD27-CD24- B cells (DN2 cells), whose expansion has been described previously in lupus patients.

\section{\begin{tabular}{|l|l}
\hline Al-09 T FOLLICULAR HELPER (TFH) CELLS ARE INCREASED IN \\
\hline
\end{tabular} ASYMPTOMATIC ANTI-NUCLEAR ANTIBODY (ANA) ${ }^{+}$ INDIVIDUALS AND APPEAR TO PLAY A ROLE IN EPITOPE SPREADING}

1,2,3,4 Joan Wither* ${ }^{1}$ Nan-Hua Chang, ${ }^{3,4,5,6}$ Sindhu R Johnson, ${ }^{3}$ Waleed Hafiz, ${ }^{1,2}$ Kieran Manion, ${ }^{1,2}$ Dario Ferri, ${ }^{1}$ Ariana Karanxha, ${ }^{1}$ Babak Noamani, 'Dennisse Bonilla, ${ }^{1}$ Sina Rusta-Sellehy, ${ }^{7}$ Larissa Lisnevskaia, ${ }^{3,4}$ Zahi Touma, ${ }^{8,9}$ Earl Silverman, ${ }^{3,4}$ Arthur Bookman, ${ }^{1}$ Carolina Landolt-Marticorena, ${ }^{1,2}$ Yuriy Baglaenko. ${ }^{1}$ Krembil Research Institute, University Health Network, Toronto, Canada; ${ }^{2}$ Department of Immunology, University of Toronto, Toronto, Canada; ${ }^{3}$ Division of Rheumatology, University Health Network, Toronto, Canada; ${ }^{4}$ Department of Medicine, University of Toronto, Toronto, Canada; ${ }^{5}$ Division of Rheumatology, Mount Sinai Hospital, Toronto, Canada; ${ }^{6}$ Institute of Health Policy, Management and Evaluation, University of Toronto, Toronto, Canada; ${ }^{7}$ Lakeridge Heath Services, Oshawa, Canada; ${ }^{8}$ Division of Rheumatology, Sick Children's Hospital, Toronto, Canada; ${ }^{9}$ Department of Pediatrics, University of Toronto, Toronto, Canada

\subsection{6/lupus-2018-Ism.9}

Background The diagnosis of Systemic Autoimmune Rheumatic Diseases (SARD), including Systemic Lupus Erythematosus (SLE), relies on the presence of ANAs, many of which can be detected years before clinical manifestations. However, ANAs are also seen in healthy individuals most of whom will not develop SARD. A number of cellular immune changes are seen in SARD, and thus could constitute potential biomarkers/ treatment targets for SARD, however it is not known at what point in disease progression these develop.

Methods Healthy $\mathrm{ANA}^{-}$controls $(n=32)$ and $\mathrm{ANA}^{+}(\geq 1: 160$ by immunofluorescence) participants with no (asymptomatic $\mathrm{ANA}^{+}, \mathrm{n}=61$ ), at least one (UCTD, $\mathrm{n}=35$ ), or meeting SARD classification criteria $(n=59)$ were recruited. Peripheral blood cellular immunological changes were assessed by flow cytometry. 
Results Consistent with previous reports, SARD patients had increased proportions of activated B cells $\left(\mathrm{CD} 86^{+}\right.$or $\left.\mathrm{CD}^{+} 5^{+}\right)$ and in the SLE patient subset there were increased proportions of plasma cells/plasmablasts, as compared to $\mathrm{ANA}^{-}$controls. SARD patients also had reduced proportions of iNKT and IFN- $\gamma$ producing cells, as well as, increased proportions of memory Tfh $\left(\mathrm{CD} 4^{+} \mathrm{CXCR} 5^{\text {hi }} \mathrm{PD} 1^{\text {hi }}\right)$ and $\mathrm{T}$ regulatory (Treg, CD $4{ }^{+} \mathrm{FOXP}^{+}{ }^{+} \mathrm{HELIOS}^{+}$) cells, especially in the SLE and Sjogren's Disease patient subsets. In asymptomatic $\mathrm{ANA}^{+}$individuals and UCTD patients, similar increases in the proportion of activated B cells, Tfh, and Treg cells, and decreases in the proportion of iNKT and IFN- $\gamma$ producing cells were seen to those in SARD. In asymptomatic $\mathrm{ANA}^{+}$individuals and SARD patients, the extent of serologic changes (number of specific ANAs detected by Bioplex ${ }^{\circledR} 2200$ ANA screening system) positively correlated with activation in the switched memory $\mathrm{B}$ cell compartment and the proportion of Tfh cells, with the later being an independent predictor of serologic status in a multivariate analysis. However, significantly elevated levels of Tfh cells could still be seen in asymptomatic $\mathrm{ANA}^{+}$individuals who lacked specific ANAs. Consistent with a role for Tfh cell in ANA production there was a strong correlation between the proportion of Tfh and plasma cells in asymptomatic $\mathrm{ANA}^{+}$individuals. In preliminary studies, the majority of Tfh cells in asymptomatic $\mathrm{ANA}^{+}$and UCTD patients were Tfh2 cells, with a trend to increased proportions of Tfh 2 cells and decreased proportions Tfh17 cells as compared to active SLE patients.

Conclusions Tfh cells appear to play an important role in the development of a positive ANA and in the epitope spreading that may accompany disease progression, and therefore constitute a promising target for treatment of early disease.

\section{Al-10 TFH CELLS AND B-CELL SELECTION}

Joe Craft*. Yale University School of Medicine, New Haven, CT USA

\subsection{6/lupus-2018-Ism.10}

Background and methods Initiation of a thymus-dependent humoral immune response requires interaction of activated $\mathrm{B}$ cells and follicular B helper $\mathrm{T}$ (Tfh) cells, a specialized subset of CD4 T helper (Th) cells. Differentiation of Tfh cells leads to their localization to $\mathrm{B}$ cell follicles and germinal centers (GC) of secondary lymphoid organs, with their expression of costimulatory proteins and secretion of cytokines promoting GC B-cell proliferation, immunoglobulin gene hypermutation, and development of B-cell memory and long-lived plasma cells. Results and conclusions The transcriptional repressor Bcl6 acts cell autonomously to drive a Tfh-cell development program, including upregulation of proteins necessary for function, with environmental signals promoting differentiation of these cells at the expense of other CD4 T-cell subsets. Tfh cells undergo differentiation within the GC, in part mediated by STAT4 and STAT6-driven chromatin remodeling, enabling differential cytokine production and consequent help to B cells, with affinity selection of the latter promoted by regulatory GC $\mathrm{T}$ cells. Therapeutically, blockade of Tfh-GC B interactions interrupts end-organ disease in the systemic autoimmune disease lupus, while resetting the Tfh-cell phenotype toward normal homeostasis.
Acknowledgements Supported by NIH AR40072 and AR068662, and the Lupus Research Alliance.

\section{Al-11 THE KINASE INHIBITORY REGION OF SUPPRESSOR OF CYTOKINE SIGNALING-1 MODULATES AUTOINFLAMMATORY SKIN PATHOLOGY}

Antia E Veal, Brandon Lam, Teresa Collins, Joseph Larkin III*. University of Florida, Department of Microbiology and Cell Science, USA

\subsection{6/lupus-2018-Ism.11}

Background Although aberrant antibody production is a lupus disease hallmark, abundant evidence implicates a dysregulated peripheral $\mathrm{T}$ lymphocyte repertoire in the onset and progression of lupus. Notably, the intracellular protein suppressor of cytokine signaling-1 (SOCS1) has been shown to regulate $\mathrm{T}$ lymphocyte effector functions and modulate lupus-like pathologies in rodent models. Significantly, peritoneal injection of a peptide (SOCS1-KIR), capable of mimicking SOCS1, was effective in mitigating $\mathrm{T}$ lymphocyte effector functions associated with lupus disease progression. Moreover, topical application of SOCS1-KIR 'eyedrops' was effective in mitigating experimental autoimmune uveitis. The peptide has been shown to function through the inhibition of the janus kinases Jak2 and Tyk2. We have previously shown that peritoneal injection of SOCS1-KIR inhibited lymphadenopathy in MRL lpr/lpr mouse model of spontaneous lupus that was correlated to decreased frequencies of interferon gamma producing memory $\mathrm{T}$ cells (Collins et al, 2018 (under revision)). In addition, the peritoneal injection of SOCS1-KIR also inhibited spontaneous lesion formation=In this study we test the hypotheses that SOCS1 modulates skin pathology and that topical application of the SOCS1-KIR peptide will have efficacy in the imiquimod induced lesion model.

Methods SOCS1 heterozygous mating pairs, sufficient and deficient of interferon gamma, were obtained from St. Jude and used to generate mice used in the experiment. Spontaneous skin lesions were assessed by histology. In addition, cytokine neutralizing antibodies were administered to evaluate mechanisms promoting lesion formation. Imiquimod was administered in the presence or absence of SOCS1-KIR to mice sufficient and deficient in SOCS1. Lesion formation was subsequently assessed.

Results SOCS1 \pm IFN gamma -/- mice, but not SOCS \pm , or IFN gamma -/- spontaneously developed epidermal hyperplasia. The SOCS1 1 , IFN gamma skin lesions were heavily infiltrated with macrophages and IL17 producing T lymphocytes. In addition, imiquimod induced skin lesions were exacerbated on SOCS1 \pm mice compared to WT. Significantly, the topical administration of SOCS1-KIR to imiquimod treated murine skin reduced epidermal hyperplasia, erythema, and scaling.

Conclusions Together these results suggest that a peptide mimic of SOCS1 may have value as a therapeutic for lupus through topical and/or systemic administration.

Acknowledgements The study was supported by a grant from the Lupus Research Institute, The national Psoriasis Foundation, a BD Biosciences Research Grant, the NIH/NCATS Clinical and Translational Science Awards to the University of Florida TL1 TR000066 and UL1TR000064, a sub-award from NIH/NIAID/U01AI101990, and the University of Florida. 